Egypt. Acad. J. biolog. Sci., 2(2): 27 - 32(2010)

Email: egyptianacademic@yahoo.com

Received: 20/6/2010
C. Physiology \& Molecular Biology

ISSN: 2090-0767

www.eajbs.eg.net

\title{
Polymerase Chain Reaction as a Rapid Tool for the Diagnosis of Pulmonary Tuberculosis
}

\section{Mogahid M Elhassan; Ahmed H Ibrahim and Miskelyemen A Elmekki}

College of Medical Laboratory Science, Sudan University of Science and Technology, Khartoum, Sudan, P O Box 407, e-mail: mogahidelhassan@yahoo.com

\begin{abstract}
Objective: This study aimed to use the polymerase chain reaction (PCR) as a rapid tool for the diagnosis of pulmonary tuberculosis from sputum samples. Clinically suspected tuberculosis patient in Khartoum state were targeted.

Materials and Methods: Sputum specimens were collected from patients attending Abu Anga Hospital, Alsha'ab Teaching Hospital and Tuberculosis Reference Laboratory. Patients were consented and informed. Sputum samples were stained with ZN stain, then decontaminated and cultured on LJ medium. Part of the sputum was used for DNA extraction by isopropanol method for PCR.

Results: $37(21.6 \%)$ smears from the collected 171 sputum samples showed positive ZN smears while 134 (78.4\%) showed negative result. On LJ medium, 23.4\% showed MTC-like colonies, 5.8\% were considered rapidly growing Mycobacteria and 70.8\% samples revealed contamination or no growth. The MTC-like colonies were confirmed by conventional methods.

When PCR was performed, 142 (83\%) samples showed a band typical in size (123 bp) to the target gene of Mycobacterium tuberculosis complex (IS 6110) as indicated by the standard DNA marker. 29(17\%) samples were negative.

Conclusion: These results revealed clearly the importance, feasibility and sensitivity of PCR as a rapid diagnostic tool to detect M. tuberculosis directly from sputum samples.
\end{abstract}

Key words: tuberculosis, IS 6110 gene, Sudan, TB diagnosis

\section{INTRODUCTION}

Tuberculosis remains a major global health problem. The actual global prevalence of Mycobacterium tuberculosis infection is $32 \%$, corresponding to approximately 1.9 billion people. According to the world health organization there were 8.8 million estimated new cases (case rate $140 / 100,000$ ) of pulmonary tuberculosis. An estimated 1.9 million people died of tuberculosis, including patients co-infected with human immunodeficiency virus (HIV) (Murray et al., 2007).

However, the diagnosis of tuberculosis continues to pose serious problems mainly because of difficulties in differentiating between patients with active tuberculosis and those with healed lesions, normal Mycobacterium bovis BCG (Bacillus Calmette Guerin) vaccinated individuals, and unvaccinated Manteux positives (Garg et al., 2003). Physicians still rely on conventional methods such as ZiehlNeelsen (ZN) staining, fluorochrome staining, sputum culture, gastric lavage, and other non-traditional methods. Tuberculosis Skin Test (TST) has been in use for the diagnosis of tuberculosis infection since 1910. TST or intradermal Manteux is the oldest diagnostic test and despite the 
diagnostic limitations it is included in the WHO latest recommendations for TB control. TST is based on a proteinpurified derivate (PPD), resulting from a culture filtrate of tubercle bacilli containing over 200 antigens common both in bacilli Chalmette-Guerin vaccine (BCG) and in most non tuberculosis bacteria. Consequently, the TST specificity is low and also the ability to distinguish latent $\mathrm{TB}$ infection is limited (Tsara et al., 2009). Although the conventional technique of direct smear examination with Ziehl-Neelsen staining $(\mathrm{ZN})$ is cheap and easy to perform; its low sensitivity is a major drawback. Depending on the number of specimens examined, $\mathrm{ZN}$ detects 30 to $60 \%$ of the culturepositive "TB suspects. Furthermore, it requires sputum samples collected on consecutive days, making the procedure slow and making patient compliance with the diagnostic process difficult (Ndugga et al., 2004).

The microbiological culture, generally used in suspected pulmonary cases and sputum smear microscopy negative cases, has the advantage of allowing the detection and isolation of the Mycobacteria, the identification of the species and/or of the isolated complex, and the determination of the sensitivity of the microorganism to chemotherapeutic agents for TB. The principal culture media used are Lowenstein-Jensen (egg-based solid medium) and Middlebrook (solid or liquid, in agar medium). Despite its importance, the culture of Mycobacterium tuberculosis is timeconsuming, due to the slow growth of the bacillus (15-20 h), and the test does not always present $100 \%$ positivity (Teixeira et al., 2007).

Several immune based tests have also been developed aiming to improve diagnostic procedures of TB infection. Serologic tests are commercially available and they are based on the detection of the antibody immune response to Mycobacterium tuberculosis. The antibody detection kits differ in their features, depending on the kind and the site of the target antigen and also on the incubation techniques. Systematic reviews, regarding antibody detection tests, have showed that they vary widely in performance and sensitivity (Tsara et al., 2009).

Molecular technique such as the polymerase chain reaction (PCR) permits the direct detection and identification of infectious agents in clinical specimens, saving days to weeks in diagnostic time. Its application to infectious disease caused by fastidious or slow growing microorganism, such as $M$. tuberculosis, has the potential to provide a truly rapid laboratory diagnosis with the attended improvement in patient management and reduction of medical costs (Amato and Miller. 2008).

Early diagnosis of the causative agent followed by adequate treatment is essential to prevent both morbidity and mortality (Ndugga et al., 2007).

The TB diagnostic techniques currently used are slow and have sensitivities and specificities that need to be improved. Although the presumptive diagnosis of TB may be obtained through clinical history and radiological findings, the final diagnosis still depends on smear and culture.

The most promising technique for a fast diagnosis is based on the polymerase chain reaction (PCR), which is theoretically capable of detecting one copy of DNA of any cell. Besides the high sensitivity and specificity, this technique can produce results in few hours, and is being used in the diagnosis of a number of infectious diseases. 
The study aimed to use polymerase chain reaction (PCR), as rapid tool for the diagnosis of pulmonary tuberculosis from sputum samples.

\section{MATERIALS AND METHODS Study Population and sampling}

One hundred and ninety four suspected patients attending Abu Anga Hospital, DOTS (Directly Observed Treatment, Short course) reference Tuberculosis Laboratory and Elasha'ab Teaching Hospital were recruited to this study.

Data were collected using a standardized questionnaire eliciting information on symptoms of tuberculosis, risk factors, social history, history of previous tuberculosis, tuberculosis treatment, and tuberculin skin testing. Sputum samples were collected in clean, wide mouthed, and leak proof specimen containers.

A direct smear was made from each sputum specimen, stained by the $\mathrm{ZN}$ method, and read at the center. After that, the specimen was sent as soon as possible (i.e., $<24$ hours) to reference Tuberculosis Laboratory for culture and DNA extraction.

Sputum samples were decontaminated before culture and DNA extraction.

Sputum samples were then cultured on LJ medium. Growth was monitored daily during the first week to observe the presence of rapid growers which if present will show growth within 7 days, and then the growth was observed weekly up to the 8 th week. Identification of Isolate was made by growth rate and biochemical reactions.

\section{Molecular Identification (PCR)}

Polymerase chain reaction was used to diagnose pulmonary tuberculosis from direct sputum samples.
DNA was extracted by Isopropanol extraction method by mixing $100 \mathrm{ul}$ of decontaminated sample in eppendorf tube $(1.5 \mathrm{ml})$ with $400 \mathrm{ul}$ of lysis buffer and $300 \mathrm{ul}$ of Isopropanol, centrifuged at 12000 rpm for 10 minutes, the supernatant was decanted by gently inverting the tube, then the pellet was washed by $75 \%$ Ethanol, and re centrifuged at $12000 \mathrm{rpm}$ for 5 minutes, the previous step was repeated three time, the final pellet was re-suspended in 50 ul Tri EDTA Amplification of insertion sequence IS6110 (123 bp) (Eisenach et al., 1990) was performed with a set of primers having the following sequence: Forward:

(CCTGCGAGCGTAGGCGTCGG)

Reverse:

(CTCGTCCAGCGCCGCTTCGG)

A master mix reagent was prepared for 100 reactions according to (Eisenach et al., 1990), $5 \mu$ l template DNA from each sample was added to the master mix. The negative control contained reaction mixture without template DNA.

A PCR program was conducted with an initial 5 minutes denaturation step at $94^{\circ} \mathrm{C}$ for one cycle followed by a repeating cycles of denaturation (2 minutes at $\left.94^{\circ} \mathrm{C}\right)$, annealing ( 2 minutes at $\left.68^{\circ} \mathrm{C}\right)$ and extension $(2$ minutes at $72^{\circ} \mathrm{C}$ ) for 25 cycles, followed by a 5 minutes of final extension at $72^{\circ} \mathrm{C}$. Then, the PCR product was visualized by UV transilluminator on $1 \%$ agarose gel.

\section{RESULTS}

Among the study population (171 patients), 69.6\% were found to be males, and $30.4 \%$ were females.

All the ages were found to be affected with tuberculosis. However, the highest frequency was found among age group $21-50$ years $(68.5 \%)$, followed by age $>50(18.2 \%)$ while the lowest frequency was among age group $<20(13.3 \%)$. 
Smear positive samples were $37(21.6 \%)$ while 134 (78.4) showed negative result.

When the 171 sputum samples were cultured on LJ medium, 23.4\% showed MTC-like colonies, 5.8\% were considered rapidly growing mycobacteria and $70.8 \%$ samples revealed contamination or no growth. The MTC-like colonies were confirmed by conventional methods.

The growth rate of the isolates ranged between 3 days and 5 weeks. Most of the isolates showed visible growth after 2 weeks. Ten out of 171 isolates were identified as rapid growers mycobacteria, while the growth rate of 40 isolates ranged between 2 to 5 weeks and they were identified as slow growers and considered belonging to MTC species.

Indirect smears from suspected MTC, rapid growers of mycobacteria were all found positive for AFB.

Cultural properties of all isolates of Mycobacterium tuberculosis complex on Lowenstein Jensen medium at $37^{\circ} \mathrm{C}$ were almost the same and all colonies were found dry, rough and buff colored.

Out of the 171 samples, 40 isolates of MTC organisms (slow growers), were positive for nitrate reduction and negative for catalase at $68^{\circ} \mathrm{C}$.

All sputum sample were extracted by Isopropanol method and were subjected to PCR, 142 samples showed a band typical in size (123 bp) to the target gene (IS 6110) as indicated by the standard DNA marker. 29 samples were negative, as shown in Fig. 1 below.

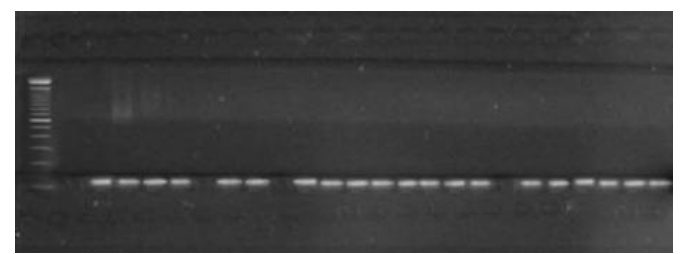

Fig. 1: The amplicon of MTC after PCR run on $1 \%$ agarose gel: Lane $1=$ marker; $2=$ negative control 2, 3, $4,5,6,8,9,11,12,13,14,15,16$, $17,18,20,21,22,23,24$ and 25 are positive for MTC (123 bp band) 7,10 and 19 are negative for MTC.

\section{DISCUSSION}

Tuberculosis is one of the leading infectious diseases in the world and is responsible for more than 2 million deaths and 8 million new cases annually (Soini and Musser, 2001). In much of the world, tuberculosis is the leading cause of death among all infectious agents, directly responsible for an estimated $7 \%$ of all deaths and $26 \%$ of all preventable deaths worldwide (McPherson and Pincus, 2006).

Because of the slow growth rate of the causative agent Mycobacterium tuberculosis, isolation, identification, and drug susceptibility testing of this organism and other clinically important mycobacteria can take several weeks or longer. During the past several years, many molecular methods have been developed for direct detection, species identification, and drug susceptibility testing of mycobacteria. These methods can potentially reduce the diagnostic time from weeks to days (Soini and Musser, 2001).

The aim of the present study was to use the polymerase chain reaction (PCR), as a rapid tool for the diagnosis of pulmonary tuberculosis from direct sputum.

Preparing DNA by using Isopropanol extraction method simplified the procedure (Not contain biohazards and take 30 minutes) so that it can be used in routine clinical practice.

Among the total of 171 specimens studied, 37(21.6\%), were smear positive, $40 \quad(23.4 \%)$ were culture positive. Smear microscopy was positive in only $12(30 \%)$ of the culture positive samples. This may be due to the low sensitivity of $\mathrm{ZN}$ stain to detect AFB which needs more than 500 bacilli $/ \mathrm{ml}$ (Iqbal, 2003). In 
comparison, PCR was positive in $95 \%$ of the culture positive. This determines the high sensitivity of PCR to detect culture positive. This finding is similar to (Kavita Modi, 2006) who found the sensitivity of smear and culture as $51 \%$ and $68 \%$ respectively. Smear microscopy was positive in only $67 \%$ of the culture positive samples while PCR was positive in $98 \%$.

Twenty (20) samples which were $\mathrm{ZN}$ positive, PCR positive but cultures negative were from patients with clinical manifestations of TB. This could be due to the presence of non-viable mycobacteria in the samples as some of the subjects were receiving anti tubercular treatment. This finding is in accordance with a report from (Luciano dos, 2003) who found that ten patients with clinical manifestations of TB were culturenegative but PCR-positive.

$84(67.4 \%)$ samples which were smear and culture negative obtained from patients clinically suspected of M. tuberculosis were PCR positive, suggesting that PCR assay is probably more sensitive than the culture by detecting non-viable and/or fewer viable organisms. Similar results were obtained by Aroma Oberoi, (2007) who found that PCR showed the highest sensitivity $(73.9 \%)$ as compared to other tests.

Two $(8.3 \%)$ culture-positive samples were PCR negative. This may indicate the presence of PCR inhibitors in these samples. This finding is similar to (Maher, 1996) who found that two culture-positive samples were PCR negative.

In conclusion, isopropanol extraction method is effective, safe and rapid procedure for DNA extraction from sputum sample.In addition, PCR is highly sensitive, effective and much more rapid than conventional methods in diagnosis of pulmonary tuberculosis.

\section{REFERENCE}

Amato, D. and Miller, A. (2008). Methods in Molecular Biology, Rapid Diagnosis of Pulmonary Tuberculosis Using Roche AMPLICOR Mycobacterium tuberculosis PCR Test Biomedical and Life Sciences,. 92: 203-214

Eisenach, K. D.; Cave, M. D.; Bates J. H. and Crawford J. T. (1990). Polymerase chain reaction amplification of a repetitive DNA sequence specific for Mycobacterium tuberculosis. J. Infect. Dis. 161: 977-981.

Garg S. K.; Tiwari, R. P.; Dileep Tiwari; Rupinder Singh; Dolly Malhotra; Ramnani, V. K.; Prasad, G.B.K.S.; Ramesh Chandra; Fraziano, M.; Colizzi, V. and Prakash S. Bisen (2003). Diagnosis of Tuberculosis; Journal of Clinical Laboratory Analysis 17:155-163

Iqbal, S.; Rizwan, I.; Mumtaz Khan; Hussain, M.; Ajaz, I. Akhtar, A., and Shabbir, I. (2003). Comparison of Two Conventional Techniques used for the Diagnosis of Tuberculosis Cases. International journal of agriculture.: 5(4): 545-547.

Kavita Modi, Parekh, Inamdar, V., Anagha, J., and Anita Kar (2006), A Comparative Study of The Diagnosis Of Pulmonary Tuberculosis Using Conventional Tools And Polymerase Chain Reaction, Indian Journal of Tuberculosis, 53: 69-76

Luciano dos, Anjos Filho, Fernanda, de Queiroz, C., Mello. Charles, G. Thornton. William, R., Leila, B., Afranio L. and Richard E. (2003). Carboxypropylbetaine and Standard N-Acetyl-L-Cysteine$\mathrm{NaOH}$ Processing of Respiratory Specimens for Increasing Tuberculosis Smear Sensitivity in Brazil. Journal of Clinical Microbiology; 40: 3219-22

Maher, M., Glennon, M., Martinazzo, G., Turchetti, E., Marcolini, S., Smith, T. and Michael T. (1996). Evaluation of a Novel PCR-Based Diagnostic Assay for Detection of 
Mycobacterium tuberculosis in Sputum Samples., Journal of Clinical Microbiology 9:23072308

McPherson R. A. and Pincus M. R. (2006). In: Henry's Clinical Diagnosis and Management by Laboratory Methods. 21st ed. W. B. Saunders Company.

Murrat, P. R., Baron, E.J., Jorgensen, J., Pfaller, M. and Landry, M. L. (2007). Manual of Clinical Microbiology, 9th Edition, American Society for Microbiology: Vol 1, p 441- 449

Ndugga K., van Cleeff M. and Juma E. (2007). Comparison of PCR with the routine procedure for diagnosis of tuberculosis in a population with high prevalences of tuberculosis and human immunodeficiency virus. $J$ Clin Microbiol 42: 1012-5.
Oberoi, A. and Aggarwal, A. (2007). Comparision of the conventional diagnostic techniques, BACTEC and PCR $J$ k Science 9(4): 179182

Soini H. and Musser J. (2001). Molecular Diagnosis of Mycobacteria; Clinical Chemistry 47: 5809-814

Teixeira I, H. C., Abramo, C. and Munk, M (2007). Immunological diagnosis of tuberculosis: problems and strategies for success Jornal Brasileiro de Pneumologia 33 (3): 323-334

Tsara, V., Serasli, E. and Christaki, V. (2009). Problems in diagnosis and treatment of tuberculosis infection. Hippokratia Medical Journal. 3(1): 20-22.

\section{ARABIC SUMMARY}

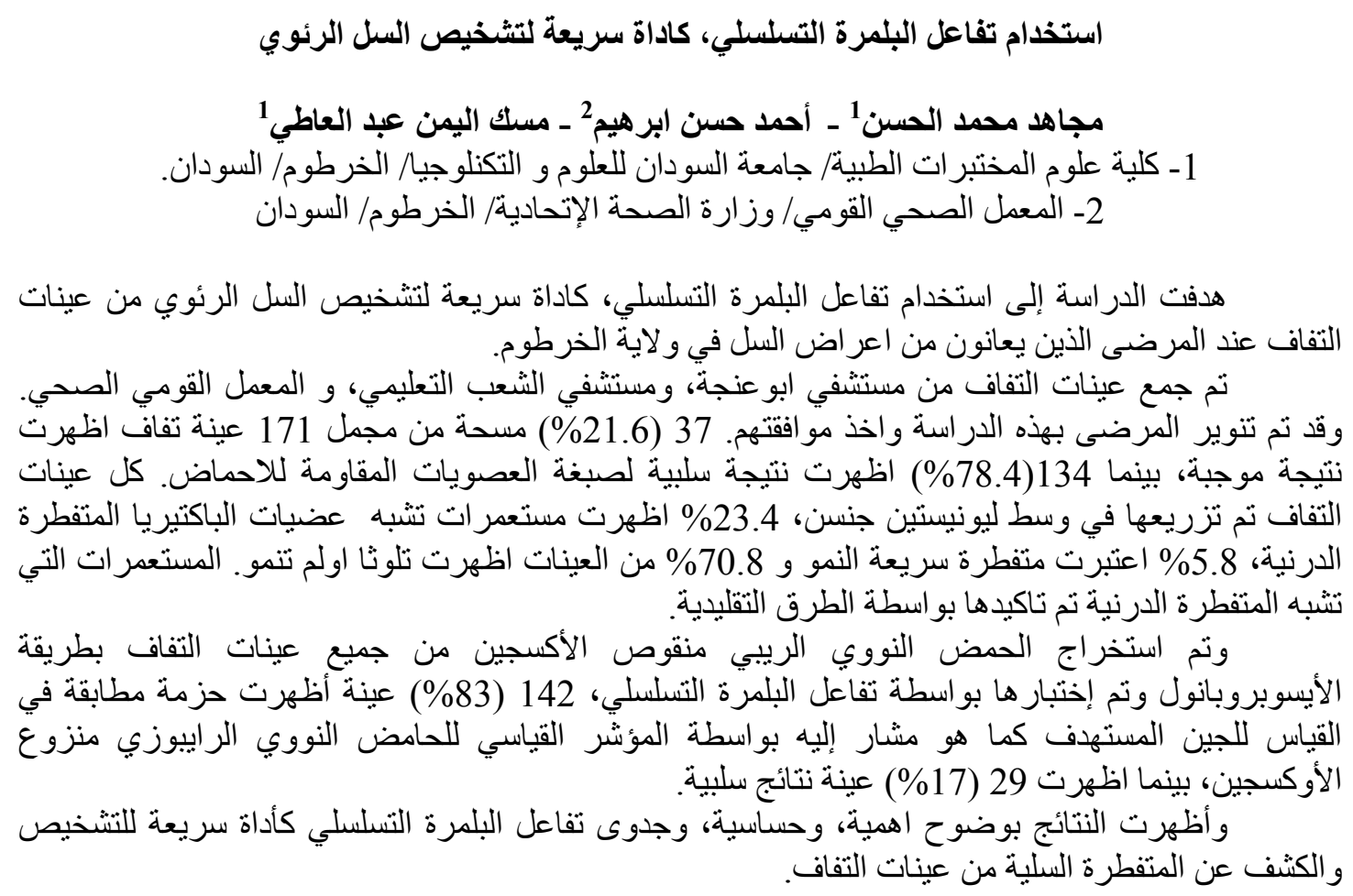

